\title{
Análisis Comparativo De Las Bibliotecas Virtuales Científicas De Salud En Los Estudiantes Universitarios
}

\author{
Saul Yasaca-Pucuna \\ Magíster en Informática Educativa \\ Técnico Docente Escuela Superior Politécnica de Chimborazo, Ecuador \\ Iván Mesias Hidalgo-Cajo \\ Máster Universitario en Ingeniería Informática: Seguridad Informática y \\ Sistemas Inteligentes \\ Docente Escuela Superior Politécnica de Chimborazo, Ecuador \\ María de los Ángeles Rodríguez-Cevallos \\ Magister en Nutrición Clínica \\ Docente Escuela Superior Politécnica de Chimborazo, Ecuador \\ Keylee Alexandra Cevallos-Paredes \\ Máster Universitario Europeo en Alimentación, Nutrición y Metabolismo \\ Docente Escuela Superior Politécnica de Chimborazo, Ecuador \\ Nelly Baltazara Latorre-Benalcázar \\ Licenciada en Ciencias de la Educación Profesora de Educación Básica \\ Docente Ministerio de Educación \\ Sulema de la Caridad Hernández-Batista \\ Especialista de Primer Grado en Medicina General Integral \\ Docente Escuela Superior Politécnica de Chimborazo, Ecuador
}

Doi:10.19044/esj.2019.v15n13p360 URL:http://dx.doi.org/10.19044/esj.2019.v15n13p360

\section{Resumen}

El objetivo de la investigación es comparar el uso y la información que se busca en las diferentes bibliotecas virtuales científicas en salud disponibles en las Instituciones de Educación Superior.

La investigación se desarrolló en las bases de datos de dos universidades de nivel superior durante el período marzo - agosto 2018, basándose en la escala Likert con 5 pesos para su valoración.

Entre los resultados de comparación de las dos bibliotecas virtuales científicas especializadas en salud se contó con la participación de 152 estudiantes de dos universidades o grupos. La investigación revela que el primer y segundo grupo casi siempre $60,90 \%$ encuentran la información a buscar y que solamente el $1 \%$ no localizan la información buscada. 
Además, con la utilización de las bibliotecas virtuales especializadas en salud nos permite confirmar que contribuye discretamente en la formación académica, y se requiere de planes de capacitación, manuales de uso para fortalecer la socialización de este recurso que es de acceso libre.

Palabras Reservadas: Bibliotecas virtuales, salud, educación superior.

\title{
Comparative Analysis of Virtual Scientific Health Libraries in Scholar Students
}

\author{
Saul Yasaca-Pucuna \\ Magíster en Informática Educativa \\ Técnico Docente Escuela Superior Politécnica de Chimborazo, Ecuador \\ Iván Mesias Hidalgo-Cajo \\ Máster Universitario en Ingeniería Informática: Seguridad Informática y \\ Sistemas Inteligentes \\ Docente Escuela Superior Politécnica de Chimborazo, Ecuador \\ María de los Ángeles Rodríguez-Cevallos \\ Magister en Nutrición Clínica \\ Docente Escuela Superior Politécnica de Chimborazo, Ecuador \\ Keylee Alexandra Cevallos-Paredes \\ Máster Universitario Europeo en Alimentación, Nutrición y Metabolismo \\ Docente Escuela Superior Politécnica de Chimborazo, Ecuador

\section{Nelly Baltazara Latorre-Benalcázar} \\ Licenciada en Ciencias de la Educación Profesora de Educación Básica \\ Docente Ministerio de Educación

\section{Sulema de la Caridad Hernández-Batista} \\ Especialista de Primer Grado en Medicina General Integral \\ Docente Escuela Superior Politécnica de Chimborazo, Ecuador
}

\begin{abstract}
The aim of this research is to compare the use and information sought in the different virtual health scientific libraries available in Higher Education Institutions.

The research was developed in the databases of two higher level universities during the period March - August 2018, based on the Likert scale with 5 pesos for its evaluation.
\end{abstract}


Among the comparison results of the two scientific virtual libraries specialized in health, 152 students from two universities or groups participated. The research reveals that the first and second group almost always $60.90 \%$ find the information to look for and that only $1 \%$ do not locate the information sought. In addition, with the use of virtual libraries specialized in health, it allows us to confirm that it contributes discreetly in academic training, and training plans and user manuals are required to strengthen the socialization of this resource that is freely accessible.

Keywords: Virtual libraries, health, higher education.

\section{INTRODUCCIÓN:}

Una biblioteca digital es una colección de objetos digitales organizados (Pérez Alarcón, 2015), que sirve a una comunidad de usuarios definida, que tiene los derechos de autor presentes y gestionados y que dispone de mecanismos de preservación y conservación. Una definición más exhaustiva propuesta en la bibliografía especializada establece que "biblioteca digital es un sistema de tratamiento técnico, acceso y transferencia de información digital, se estructura mediante una colección de documentos digitales, sobre los cuales se ofrecen servicios interactivos de valor añadido para el usuario final" (Tramullas, 2014).

Se refiere a los recursos informáticos documentales, a los que se accede mediante diferentes dispositivos de comunicación que usan los servicios de Internet. En el concepto de biblioteca digital está implícita la integración de la tecnología informática y las comunicaciones. La información ofrecida suele estar disponible en formatos pdf, doc, jpg, BMP o MP3, entre otros.

La biblioteca digital es un recinto informativo de nueva generación y se basa en las nuevas tecnologías. Las diferencias se manifiestan en el sentido de que el recinto anterior se basaba en procedimientos mecánicos y este nuevo ya utiliza procesos automatizados y, lo más importante, se puede tener acceso a todas las fuentes documentales a distancia a través de internet. Otra diferencia importante consiste en que a este recinto informático digital se puede ingresar a cualquier hora los 365 días del año. También cumple con las normas ISO y con las leyes referentes a derecho de autor, de patentes y marca (Marcum, 2015).

\subsection{Justificación/Problema}

En las bibliotecas virtuales de las Instituciones de Educación Superior así como la Escuela Superior Politécnica de Chimborazo (ESPOCH), http://bibliotecas.espoch.edu.ec/bdatos.html y de la Universidad Nacional de Chimborazo (UNACH), http://www.unach.edu.ec/bvirtual/index.php/basesde-datos-cientificas, existen una gran diversidad de bases de datos científicas 
especializadas en salud así como catálogos bibliográficos que se pueden acceder, pero ante la presencia de falta de conocimiento apropiado de acceso y uso por parte de los diferentes usuarios como son: investigadores, docentes, estudiantes y personal externo a las misma, se puede recurrir a estos recursos informáticos, así como la falta de motivación para utilizar los distintos métodos de búsqueda e inexistencias de un servicio de bibliotecas virtuales se acceso libre desde lugares externos a las universidades debido a que solo se permite el acceso con su usuario y contraseña, para acceder de manera libre y completa la visualización de documentos y descarga de los mismos, la resistencia de docentes que en su mayoría utiliza el sistema de enseñanza tradicional.

De esta manera conlleva a la incertidumbre si estos recursos están contribuyendo positivamente a la formación de los estudiantes en las instituciones de Educación Superior, además se evidencia la inexistencia de herramientas confiables de evaluación y que se ejecuten bajo políticas o estándares internacionales que facilite la toma de decisiones. Por lo que se crea un prototipo de encuesta que intente recoger un conjunto de indicadores para evaluar la efectividad de las bibliotecas virtuales especializadas en salud y sobre todo reconocer el aporte en la formación de los estudiantes.

\subsection{Revisión de la literatura}

La Biblioteca Virtual en Salud del Ecuador (BVS-Ecu) constituye una línea de trabajo estratégica del Ministerio de Salud Pública (MSP) coordinada por la Dirección Nacional de Inteligencia de la Salud, que forma parte del Centro Latinoamericano y del Caribe de Información en Ciencias de la Salud (BIREME). La BVS-Ecu es un espacio integrador para el acceso a un conjunto de recursos de información especializados en Ciencias de la Salud, disponibles en los ámbitos nacional e internacional, con el fin de convertir la Información Científica-Técnica en un componente esencial para la toma de decisiones en salud del Ecuador (Ministerio de Salud Pública, 2017).

A pesar de que existen varios modelos, metodologías, tutoriales y guías, para diseñar e implementar servicios y productos para la web. En su mayoría se basa en propuestas de la arquitectura de la información y la usabilidad, donde se indican los pasos que van desde el estudio de la comunidad de usuarios hasta la implementación tecnológica, sumándose a esto la organización de la información y el diseño gráfico de la interfaz (CÁRDENAS FRANCO, 2016).

En el artículo de investigación "Un modelo de evaluación de la calidad de las bibliotecas universitarias digitales basado en técnicas difusas", presenta un modelo que contiene tanto criterios objetivos como subjetivos, que pueden ser ponderados, que están orientados a usuarios y que usan técnicas difusas lingüísticas para obtener los valores de calidad. Dividido en: 
Un esquema de evaluación: Contiene los indicadores de calidad, tanto los objetivos como los subjetivos. Este entorno contempla el primer nivel y el segundo nivel, a su vez el segundo nivel se subdivide en 4 categorías.

Para nuestro estudio se considera este esquema de evaluación porque está compuesto de criterios cuantitativos y subjetivos, en especial atención a aquellos que sirven para observar la conducta de los usuarios o bien para recoger sus opiniones o juicios de valor. Del Primer nivel - objetivos: se selecciona el indicador (acceso a las bases de datos, consultas y para medir la visibilidad externa de la biblioteca). Del Segundo nivel - subjetivos: obtenemos directamente de las opiniones proporcionadas por los usuarios sobre el funcionamiento del sistema. De la categoría Intrínseca: se mide la calidad de precisión de las bibliotecas universitarias en términos de si los usuarios encuentran o no lo que buscan, por lo tanto, se define el indicador cualitativo (encuentra los que busca). De la categoría Contextual: para evaluar aspectos informativos desde una perspectiva de la tarea se considera los indicadores (grado de satisfacción, presenta utilidades de valor añadido, cobertura sobre la materia que se busca entre otras). De la categoría Calidad de representación: se recoge los siguientes criterios (la comprensibilidad de la biblioteca digital y la información recibida) (Herrera-Viedma, Alonso, Gijón, \& Áv, 2006)

En términos históricos el concepto de biblioteca ha acompañado a las tecnologías y ha estado muy condicionado por las mismas. Así las primeras bibliotecas en la antigüedad estaban formadas por colecciones de tablillas de arcilla, posteriormente de rollos de papiro en el antiguo Egipto, Grecia y Roma. En la Edad Media comenzó a utilizarse el pergamino como nuevo formato, hasta la invención de la imprenta en el siglo XV. El aumento de producción de libros impresos permitió la aparición de las Bibliotecas Públicas, con el objetivo de acercar la cultura a toda la sociedad a través de los libros.

A finales del siglo $\mathrm{XX}$, el avance de la tecnología y la expansión de Internet dieron lugar a la aparición de bibliotecas electrónicas, virtuales y digitales. Aunque estos términos se utilizan a menudo de forma indistinta (Hípola, Vargas-Quesada, \& Senso Ruiz, 2010), no deben confundirse:

Biblioteca tradicional: La definición de biblioteca tradicional o clásica como su nombre indica, se reserva a las bibliotecas que realizan sus procesos sin el empleo, o con muy poco empleo, de las tecnologías de información y comunicación. Se distinguen por poseer los contenidos en soporte papel y el acceso ocurre mediante referencias bibliográficas contenidas en los catálogos.

Biblioteca electrónica: Es aquella que permite acceder a bancos de información en formato electrónico, cuenta con catálogos automatizados de bibliotecas tradicionales y los usuarios pueden reproducir la producción impresa, pero con un medio diferente del soporte papel. Cuenta con un espacio 
físico donde los usuarios pueden acceder a recursos impresos y digitales. La automatización del sistema de búsqueda de información es una de sus características. Realmente no es electrónica, correspondería a una biblioteca informatizada o automatizada.

Biblioteca híbrida: Es aquella biblioteca que está en un periodo de transición hacia la biblioteca digital. Es un lugar donde coexiste el formato impreso y digital, y por lo tanto pueden existir servicios tradicionales y servicios en ambiente digital. Mantiene el espacio físico, sin cambiar la estructura de la biblioteca. Actualmente, muchas bibliotecas serían híbridas, bibliotecas con colecciones y servicios tradicionales, y con parte de su colección en formato digital. El concepto de biblioteca híbrida apareció en la bibliografía a finales de la década de 1990 (Rusbridge, 2008), pero ha caído en desuso, y en la actualidad se entiende que la evolución de las bibliotecas ya ha incorporado este modelo.

Biblioteca digital: La biblioteca digital es un conjunto organizado de documentos digitalizados, documentos que tienen un original en papel y que por medio de un proceso de escaneo se llevan a formato digital. Es una biblioteca en la que una proporción significativa de los recursos de información se encuentran disponibles en formato digital accesible por medio de las computadoras. Proporcionan sus servicios por medio de una red de computadoras, sea privada de una organización o una red de acceso público como Internet. Las bibliotecas digitales se conforman con colecciones especializadas en una temática o rama específica del conocimiento, y se diseñan en consideración a los usuarios de una organización concreta, con independencia de que cualquier usuario conectado a la red pueda acceder a ella. Una biblioteca digital puede tener documentos que constituyan versiones digitales de documentos en formato impreso. En la mayoría de los casos, el original en papel carece de importancia, debido a que la interacción del usuario con el sistema (biblioteca) se produce por medio de las redes telemáticas y no de forma física. En una biblioteca digital, los recursos de información pueden ser propios de la biblioteca o pueden distribuirse en distintas partes de la red. Los servicios están basados sobre información propia de una institución, tanto generada en esta como producto de la digitalización de colecciones impresas existentes en su fondo bibliográfico.

Biblioteca virtual: La biblioteca virtual colecciona datos electrónicos, los ordena y los ofrece al lector. La biblioteca virtual cuenta con todos los servicios necesarios de forma remota y coloca a disposición de los usuarios, servicios tradicionales y herramientas adecuadas a sus particularidades. Satisface las necesidades de información y comunicación; el espacio físico no está unido a la posibilidad de mantener una colección actualizada con amplia cobertura temática. Es accesible mediante Internet y no existe físicamente en 
ningún lugar, no tiene colección impresa ni edificio, y se crea a partir de documentos digitalizados y sitios.

En definitiva, se habló de biblioteca electrónica cuando se automatizaron determinados procesos y servicios, o de biblioteca digital cuando las colecciones de documentos comenzaron a soportarse en formato digital. Se llega al concepto de biblioteca virtual cuando las colecciones y servicios bibliotecarios en sus diversas modalidades comenzaron a integrarse en un espacio en red.

\section{Modelo de evaluación de calidad de bibliotecas universitarias}

En el artículo de investigación "Un modelo de evaluación de la calidad de las bibliotecas universitarias digitales basado en técnicas difusas" (HerreraViedma, Alonso, Gijón, \& Áv, 2006), presenta un modelo que contiene tanto criterios objetivos como subjetivos, que pueden ser ponderados, que están orientados a usuarios y que usan técnicas difusas lingüísticas para obtener los valores de calidad.

Un esquema de evaluación contiene los indicadores de calidad, tanto los objetivos como los subjetivos. Este entorno contempla el primer nivel y el segundo nivel, a su vez el segundo nivel se subdivide en 4 categorías.

Para nuestro estudio se considera este esquema de evaluación porque está compuesto de criterios cuantitativos y subjetivos, en especial atención a aquellos que sirven para observar la conducta de los usuarios o bien para recoger sus opiniones o juicios de valor. Del Primer nivel - objetivos: se selecciona el indicador (acceso a las bases de datos, consultas y para medir la visibilidad externa de la biblioteca). Del Segundo nivel - subjetivos: obtenemos directamente de las opiniones proporcionadas por los usuarios sobre el funcionamiento del sistema. De la categoría Intrínseca: se mide la calidad de precisión de las bibliotecas universitarias en términos de si los usuarios encuentran o no lo que buscan, por lo tanto, se define el indicador cualitativo. De la categoría Contextual: para evaluar aspectos informativos desde una perspectiva de la tarea se considera los indicadores (grado de satisfacción, presenta utilidades de valor añadido, cobertura sobre la materia que se busca entre otras). De la categoría Calidad de representación: se recoge los siguientes criterios (la comprensibilidad de la biblioteca digital y la información recibida).

Por lo que refiere a la evaluación de biblioteca virtuales en este trabajo, se aborda suspicazmente los indicadores calidad (que se caracteriza por la importancia del contenido), la cobertura o visibilidad de documentos, a excepción del indicador impacto (influencia de la publicación) que bien podría ser evaluados técnicamente en base al número de citas, sin embargo, se deja como inquietud para análisis en otro apartado en mejor detalle a través de los indicadores de Bibliometrics (Huamaní , y otros, 2014). 
Un aspecto importante para mencionar es que el auge de este tipo de bibliotecas no es nuevo en la comunidad educativa y que cualquier modelo de evaluación no contribuye a una aproximación valida, sino que es necesaria. por lo que este trabajo pretende recoger en su mayoría un conjunto de indicadores para evaluar este tipo de recursos bibliográficos. Aunque en realidad, una completa evaluación debe considerar los costos y beneficios, estos no serán tratados, debido a que la presente propuesta tiene como objetivo principal la aceptación y formación de los estudiantes.

\subsection{Propósito}

- Comparar las diferentes bibliotecas virtuales científicas de acuerdo frecuencia de acceso, actualización del contenido que buscan los usuarios, disponibilidad de información, pertinencia, tiempos de respuesta, actualización de la información y precisión.

\subsection{Hipótesis}

Las bibliotecas virtuales científicas de salud inciden positivamente en la formación de académica de los estudiantes universitarios.

\section{MÉTODO}

Se realizó un estudio descriptivo durante el periodo académico abril agosto 2018. Para lo cual se aplicó una encuesta vía online considerando los criterios y subcriterios para proceder a evaluar las bibliotecas virtuales, para el modelo de evaluación se tomó como base el trabajo de evaluación de la calidad de las bibliotecas universitarias digitales basado en técnicas difusas. Además, se consideran los criterios y subcriterios del estudio sobre Biblioteca virtual en Salud (Técnicas de análisis estadístico en ciencias sociales, 2015).

La población de estudio estuvo compuesta por 152 estudiantes elegidos al azar de 2 universidades o grupos, con un total de 83 estudiantes pertenecientes de la Facultad de Salud Pública (ESPOCH), mientras que los 69 corresponden a la Facultad de Ciencias de la Salud (UNACH), se seleccionaron estudiantes de estas facultades porque pertenecen a la misma área de conocimiento y el interés principal en este caso son temas en salud.

\subsection{Instrumentos}

- Un cuestionario encuesta

- Formularios Google drive.

- Microsoft Office Excel 2016, Complemento EzAnalyze

- Software estadístico JMP 


\subsection{Procedimiento}

El primer paso fue estructurar una encuesta en base a la metodología para evaluar las bibliotecas virtuales mencionadas anteriormente.

Las preguntas de la encuesta fueron planteadas en base a la escala Likert con 5 pesos para su valoración (Tabla 1), la encuesta constó de 11 ítems y dos de ellos con 4 cada uno, acoplada en torno a los criterios e indicadores de la investigación.

Tabla 1. Pesos para la valoración de encuestas de las bibliotecas virtuales de salud de las IES

\begin{tabular}{|c|c|c|}
\hline Escala & Interpretación & Peso \\
\hline Totalmente de acuerdo & Aceptación total & 5 \\
\hline De acuerdo & Conformidad & 4 \\
\hline Ni de acuerdo ni en desacuerdo & Indeciso & 3 \\
\hline En desacuerdo & Desconformidad & 2 \\
\hline Totalmente en desacuerdo & Rechazo total & 1 \\
\hline
\end{tabular}

Una vez realizada la encuesta, se diseñaron y publicaron en la plataforma Google Drive, para la recogida de datos.

Se procedió a aplicar la encuesta en los estudiantes de los dos centros educativos en el mismo momento de la investigación, Para la tabulación y análisis de los resultados de cada encuesta se utilizó el complemento EzAnalyze como herramienta de procesamiento de datos, mientras que para la valoración global se utilizó el estadístico T-Student para una muestra (Técnicas de análisis estadístico en ciencias sociales, 2015). Finalmente, para analizar la hipótesis se importa la base de datos producto de la encuesta en el software estadístico JMP.

\section{Resultados}

Para esta sección se exponen los resultados más relevantes de cada grupo:

- Opinión de los usuarios sobre frecuencia de visita a las Bibliotecas virtuales.

Tabla 2. Criterio sobre la frecuencia de acceso a la biblioteca virtual

\begin{tabular}{|c|c|r|r|r|}
\hline Ítem & Opciones & ESPOCH & UNACH & \multicolumn{1}{c|}{ Media } \\
\hline \multirow{4}{*}{$\begin{array}{c}\text { ¿Con qué } \\
\text { frecuencia accede a } \\
\text { la biblioteca } \\
\text { virtual? }\end{array}$} & 1 a 2 días a la semana & $23,30 \%$ & $37,90 \%$ & $30,60 \%$ \\
\cline { 2 - 5 } & 3 a 7 días a la semana & $14,20 \%$ & $5,70 \%$ & $9,95 \%$ \\
\cline { 2 - 5 } & Una vez al mes & $46,30 \%$ & $48,63 \%$ & $47,47 \%$ \\
\cline { 2 - 5 } & Cada 3 meses & $8,62 \%$ & $3,49 \%$ & $6,06 \%$ \\
\cline { 2 - 5 } & Nunca ha utilizado & $7,58 \%$ & $4,28 \%$ & $5,93 \%$ \\
\hline \multicolumn{2}{|c}{ Total } & $100,00 \%$ & $100,00 \%$ & $100,00 \%$ \\
\hline
\end{tabular}

La Tabla 2., ilustra que para el primer grupo en su mayoría el 46,30\% acceden a las bibliotecas virtuales con una frecuencia de una vez al mes, y el 
segundo grupo se obtiene una media del 48,63\%, y una media de $47.47 \%$ para los dos grupos.

- Opinión de los usuarios sobre si encuentran la información que buscaba.

Tabla 3. Criterio sobre si encontró la información que buscaba en la biblioteca virtual

\begin{tabular}{|c|c|r|r|r|}
\hline Ítem & Opciones & ESPOCH & UNACH & \multicolumn{1}{c|}{ Media } \\
\hline \multirow{4}{*}{$\begin{array}{c}\text { ¿Encontró la información } \\
\text { que buscaba? }\end{array}$} & Siempre & $9,10 \%$ & $9,20 \%$ & $9,15 \%$ \\
\cline { 2 - 5 } & Casi siempre & $65,60 \%$ & $56,20 \%$ & $60,90 \%$ \\
\cline { 2 - 5 } & Algunas veces & $22,90 \%$ & $31,30 \%$ & $27,10 \%$ \\
\cline { 2 - 5 } & Rara vez & $1,50 \%$ & $2,00 \%$ & $1,75 \%$ \\
\cline { 2 - 5 } & Nunca & $0,90 \%$ & $1,10 \%$ & $1,00 \%$ \\
\hline Total & & $100,00 \%$ & $100 \%$ & $99,90 \%$ \\
\hline
\end{tabular}

La Tabla 3., ilustra que para el primer grupo obtiene el 65,60\% encuentran la información que buscaban, mientras que para el segundo grupo con un valor de $56,20 \%$, reflejando un promedio de $60.90 \%$ para ambos grupos.

Información bibliotecas virtuales especializadas en salud ESPOCH - UNACH

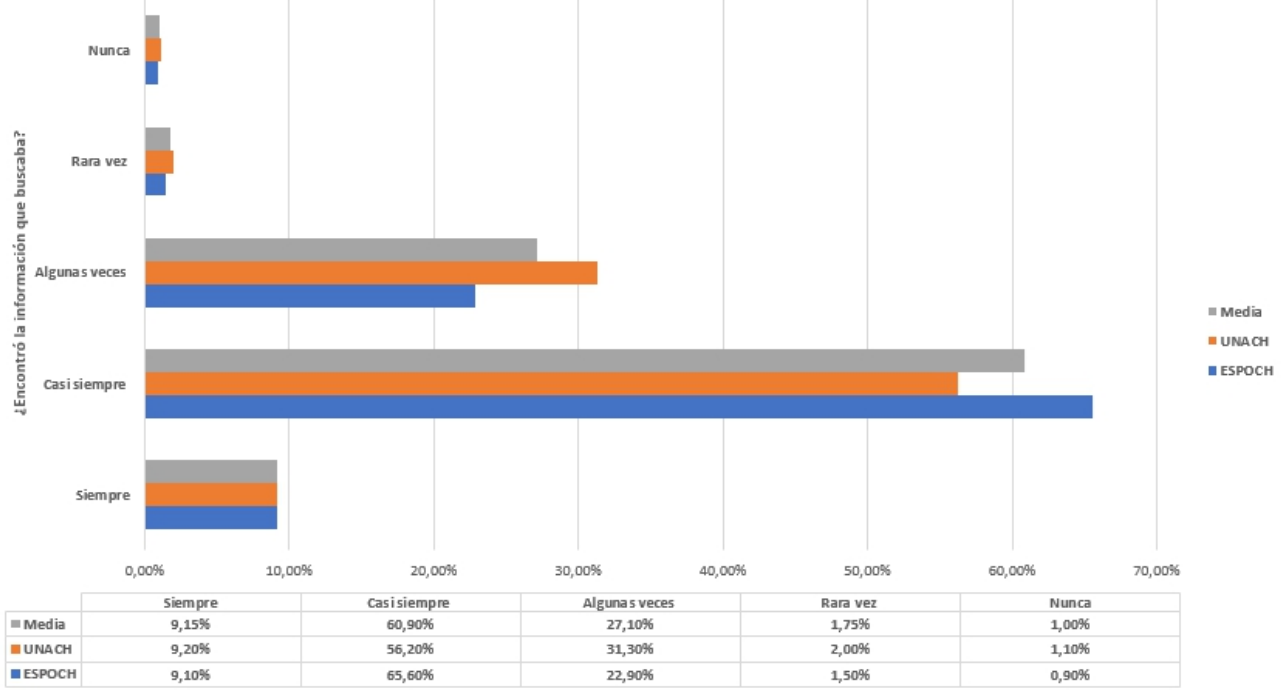

Figura 1. Criterio sobre disponibilidad de la información de Bibliotecas virtuales especializadas en salud de las IES

De acuerdo con la Figura 1., ambos grupos Casi siempre encuentran la información a buscar así se obtiene que la UNACH 56,20\% frente a la ESPOCH con $65,60 \%$ encuentran la información que buscaban, con un promedio de $60.90 \%$ para ambos grupos y no obtienen la información solicitada la UNACH $1,10 \%$ y la ESPOCH $0,90 \%$ con un promedio de 1,00 $\%$ no encuentran la información que buscaban. 
- Opinión acerca de la documentación que ofrece como respuesta la opción REALIZAR BÚSQUEDA, según los criterios "tiempo de respuesta, precisión, pertinencia y la documentación se encuentra actualizados; se evalúan con los siguientes pesos de calificación: Muy buenos (5), Buenos (4), Ni bueno ni malo (3), Malos (2) y Muy malos (1)

Tabla 4. Criterios sobre pertinencia, tiempos de respuesta, actualización de la información y precisión.

\begin{tabular}{|c|c|c|c|c|}
\hline Ítem & Opciones & ESPOCH & UNACH & Media \\
\hline \multirow{6}{*}{$\begin{array}{c}\text { Pertinencia o relación con } \\
\text { el tema }\end{array}$} & Muy buenos & $7,80 \%$ & $13,50 \%$ & $10,65 \%$ \\
\hline & Buenos & $52,30 \%$ & $63,60 \%$ & $57,95 \%$ \\
\hline & Ni bueno ni malo & $29,70 \%$ & $19,20 \%$ & $24,45 \%$ \\
\hline & Malos & $4,00 \%$ & $2,80 \%$ & $3,40 \%$ \\
\hline & Muy Malos & $6,20 \%$ & $0,90 \%$ & $3,55 \%$ \\
\hline & Total & $100,00 \%$ & $100,00 \%$ & $100,00 \%$ \\
\hline \multirow{6}{*}{ Tiempos de respuesta } & Muy buenos & $12,30 \%$ & $9,40 \%$ & $10,85 \%$ \\
\hline & Buenos & $48,20 \%$ & $57,10 \%$ & $52,65 \%$ \\
\hline & Ni bueno ni malo & $35,80 \%$ & $31,60 \%$ & $33,70 \%$ \\
\hline & Malos & $2,30 \%$ & $1,30 \%$ & $1,80 \%$ \\
\hline & Muy Malos & $1,40 \%$ & $0,60 \%$ & $1,00 \%$ \\
\hline & Total & $100,00 \%$ & $100,00 \%$ & $100,00 \%$ \\
\hline \multirow{6}{*}{$\begin{array}{l}\text { Los datos o documentos } \\
\text { están siempre } \\
\text { actualizados }\end{array}$} & Muy buenos & $13,70 \%$ & $14,60 \%$ & $14,15 \%$ \\
\hline & Buenos & $47,10 \%$ & $63,60 \%$ & $55,35 \%$ \\
\hline & Ni bueno ni malo & $31,90 \%$ & $18,40 \%$ & $25,15 \%$ \\
\hline & Malos & $5,70 \%$ & $2,60 \%$ & $4,15 \%$ \\
\hline & Muy Malos & $1,60 \%$ & $0,80 \%$ & $1,20 \%$ \\
\hline & Total & $100,00 \%$ & $100,00 \%$ & $100,00 \%$ \\
\hline \multirow{6}{*}{ Precisión } & Muy buenos & $7,40 \%$ & $12,30 \%$ & $9,85 \%$ \\
\hline & Buenos & $51,60 \%$ & $63,40 \%$ & $57,50 \%$ \\
\hline & Ni bueno ni malo & $31,50 \%$ & $21,80 \%$ & $26,65 \%$ \\
\hline & Malos & $6,20 \%$ & $2,40 \%$ & $4,30 \%$ \\
\hline & Muy Malos & $3,30 \%$ & $0,10 \%$ & $1,70 \%$ \\
\hline & Total & $100,00 \%$ & $100,00 \%$ & $100,00 \%$ \\
\hline
\end{tabular}

La Tabla 4. y Figura 2., ilustra la información sobre Pertinencia o relación con el tema: para el primer grupo el $52.30 \%$ en cuanto al criterio pertinencia o relación con el tema son buenos, mientras que para el segundo grupo se obtiene el 63,60\%, y una media 57,95\% para ambos grupos. Tiempos de respuesta: para el primer grupo en la mayoría el 48,20\% al realizar la búsqueda los tiempos de respuesta son buenos, mientras que para el segundo grupo se obtiene el 57,10\%, y media total de 52,65\% de ambos grupos. Los datos o documentos están siempre actualizados: para el primer grupo el $47,10 \%$ en cuanto al criterio de actualización son buenos, mientras que para el 
segundo grupo se obtiene el 63,60\%, y una media total de 55,35\%. Precisión: para el primer grupo se obtiene mayoritariamente el 51,60\% que al realizar la búsqueda en cuanto al criterio precisión son buenos, mientras que para el segundo grupo se obtiene un valor del $63,40 \%$, y una media total de $57,50 \%$. criterios sobre pertinencia, tiempos de respuesta, actualización de la información y precisión

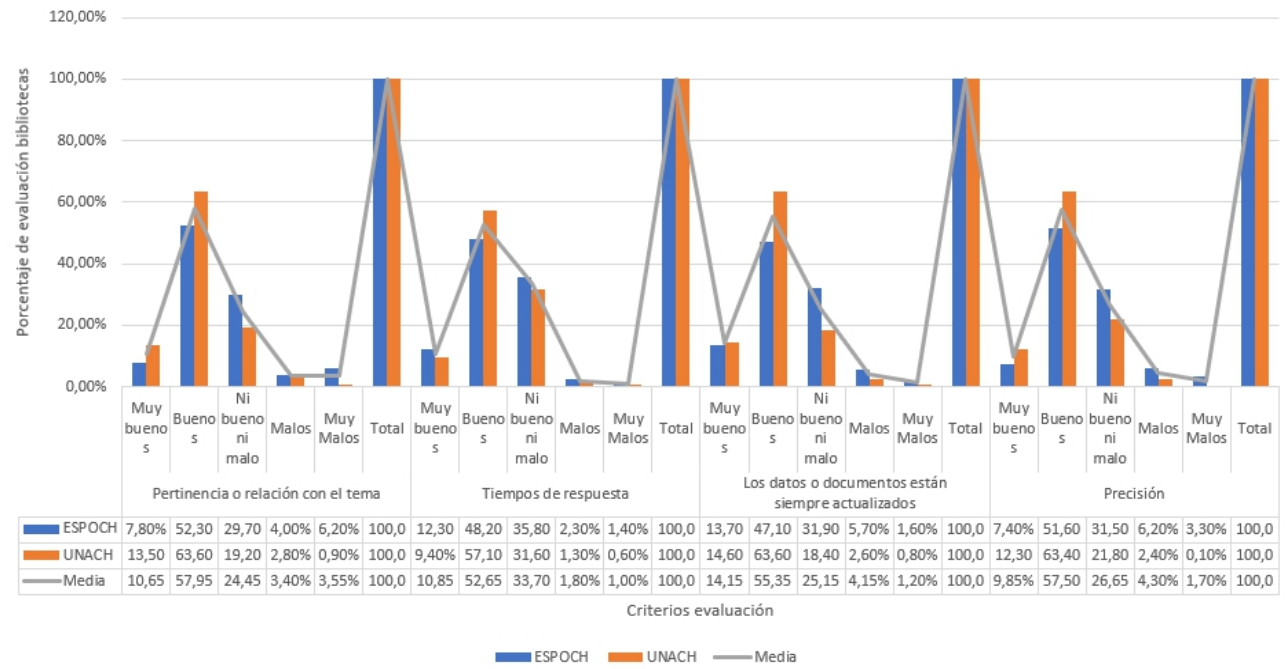

Figura 2. Criterios sobre pertinencia, tiempos de respuesta, actualización de la información y precisión

Para la comprobación de la hipótesis se utilizó los datos de los diferentes indicadores que son el resultado de aplicar la encuesta. En primera instancia se realizó el estadístico T-student para una muestra, para lo cual se debió verificar la normalidad, pero no se cumple este parámetro debido a la dispersión de los datos tal como se refleja en la Figura 3.

Figura 3. Prueba de Normalidad

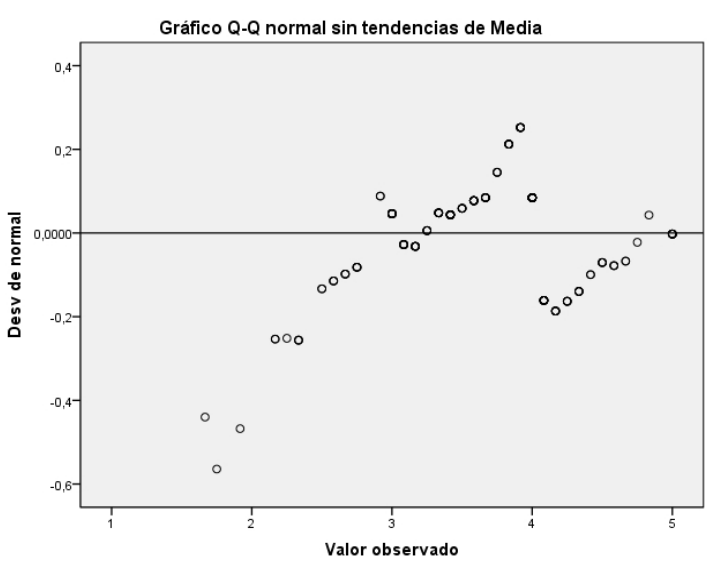




\section{Conclusión:}

- Para la estructuración del instrumento de recolección de datos se considera modelos de evaluación de calidad en bibliotecas universitarias digitales a través de sus esquemas y niveles, así como también guías y diferentes criterios.

- Con respecto a la frecuencia de acceso a la biblioteca virtual el primer grupo en su mayoría el $46,30 \%$ acceden a las bibliotecas virtuales con una frecuencia de una vez al mes, y el segundo grupo se obtiene una media del $48,63 \%$

- Se menciona que la ESPOCH con $65,60 \%$ frente a la UNACH con el $56,20 \%$ encuentran la información que buscaban, con un promedio de $60.90 \%$ para ambos grupos.

- No se obtiene la información solicitada la UNACH con el $1,10 \%$ y la ESPOCH con $0,90 \%$ en un promedio de $1,00 \%$ no encuentran la información que buscaban.

- El empleo de una metodología estandarizada proporciona un marco de trabajo sistemático que facilita las tareas de análisis

- Cabe mencionar que en el presente trabajo se recoge ciertos indicadores de impacto o influencia de la publicación que pueden ser evaluados a través de indicadores Bibliometrics, sin embargo, como trabajo futuro se podrá realizar un seguimiento a la producción local en este tipo de bibliotecas.

\section{References:}

1. Tramullas, J. (2014). Propuestas de concepto y definición de la biblioteca digital. Madrid.

2. CÁRDENAS FRANCO, L. (2016). PROPUESTA DE DISEÑO DE UN MODELO DE GESTIÓN DE PROYECTOS WEB DESDE LA METODOLOGÍA DE DISEÑO CENTRADO EN EL USUARIO PARA EL SISTEMA DE PORTALES DE LA UNIVERSIDAD EAFIT. Medellín.

3. Herrera-Viedma, E., Alonso, S., Gijón, J., \& Áv, B. (2006). Un modelo de evaluación de la calidad de las bibliotecas universitarias digitales basado en técnicas difusas. Revista electrónica de biblioteconomía e ciencia sa informacao.

4. Hípola, P., Vargas-Quesada, B., \& Senso Ruiz, J. (2010). Bibliotecas digitales: situación actual y problemas. El profesional de la información, 4-13.

5. Huamaní, C., Romaní, F., González Alcaide, G., Mejía, M., Ramos, J., Espinoza, M., \& Cabezas, C. (2014). Colaboración Sudamericana en publicaciones científicas sobre leishmaniasis: análisis bibliométrico en SCOPUS (2000-2011). Sao Paulo. 
6. Marcum, D. (2015). Boletín de la Asociación Andaluza de Bibliotecarios. Andaluza.

7. Ministerio de Salud Pública. (2017). Biblioteca Virtual en Salud. Obtenido de http://bvs-ecuador.bvsalud.org/

8. Pérez Alarcón, A. (2015). Biblioteca Digital. Barcelona: BIBLIODOC.

9. Ramírez Céspedes, Z. (2006). Criterio e indicadores para evaluar las bibliotecas digitales. La Habana: Acimed.

10. Rusbridge, C. (2008). Towards the Hybrid Library. D-Lib Magazine.

11. Saracevic, T., \& Covi, L. (2010). Challenges for Digital Library Evaluation.

12. Técnicas de análisis estadístico en ciencias sociales. (2015). Editorial Complutense. 